\title{
Design of Motion Control System for Quadruped Robot
}

\author{
Caixia WANG ${ }^{a}$, Zhiwen ZHOU ${ }^{b}$ and Ling GUO $^{c}$ \\ College of Electrical Engineering, Northwest University for Nationalities, Lanzhou, 730124, China \\ awangcaixia1@163.com, bIxzzw407@163.com, '78493218@qq.com
}

\section{Keywords: Quadruped Robot, PWM, Steering Gear, Stability}

\begin{abstract}
Quadruped robots process lower control complexity and structural redundancy compared with 6 or 8 feet robots. Quadruped robots can adapt to all kinds of known or unknown complex environment, such as in disaster rescue, aerospace, space exploration, pipeline surveying and exploration. It can meet all kinds of requirements. In this paper, master control chip ATMEGA16 is applied to produce certain types of PWM signal to control the steering gear and realize motion control of each joint of the quadruped robot. On the premise of guarantee the stability of the robot, a variety of common posture movements including lying down, walk, the trot, the gallop at low speed etc. are achieved.
\end{abstract}

\section{Introduction}

A quadruped robot system is complex in structure and process high redundancy, high flexibility, strong adaptability. It is able to crawl in desert, swamp as well as working in the hills or the nonplanar environment. With their feet as supporting roles, quadruped robot can easily get through various complex geological areas. With the rapid development of science and technology in recent years, the research of quadruped robot has been greatly improved not only in the enhancement in functions but also in terms of load and speed. "TEKKEN" robot developed by Japan is a typical representative of quadruped robot. "TEKKEN" can achieve stable walking. At the same time it also can step over obstacle in the height of $4 \mathrm{~cm}$, climb stairs with gradient $12^{\circ}$ and walk across complex geological area [1]. It can achieve speeds of up to $0.8 \mathrm{M} / \mathrm{S}$ which is the same as people walking speed under the condition of load [2].

Based on the analysis of structure of quadruped robot, stable condition and most basic conditions for the robot robot walking stable are determined. With the application of master control chip ATMEGA16 and simulation of quadruped robot gait types, reasonable parameters of gait are determined and recorded by changing the angle of each joint of the robot. Comprehensive operation of quadruped robot is realized by changing the frequency and order of the stable data.

\section{Model of Quadruped Robot}

When walking, to ensure stable quadruped robot will take at least three legs as support while another leg swings to achieve all sorts of movements such as moving forward, backward, left, right and around[3]. The raising and putting down of legs must be operated according to certain sequence so that the barycenter of robot lies in the triangle composed by the feet on the plane and the motion stable is ensured.

The simplified structure of a quadruped robot is shown in figure 1. It is a kind of robot with two joints on each leg and can move in a plane [4].The following figures shows models of quadruped robot in different working modes.

All sorts of movements are realized by the switching of the four legs. Figure 1(a) shows the model structure of quadruped robot. The working area of the quadruped robot can be divided into four parts named as I, II, III and IV when the neighboring legs are connected. According to the location of the center of gravity, the moving direction is decided. From Figure 1(a), it is known that the center of gravity can locate in area I, II, III and IV. No matter which area the center of gravity is located, the quadruped robot must balance its center of gravity by switching its legs. The motion model structure 
of quadruped robot is shown in Figure 1(b). There are 24 kinds of switching modes to ascertain the stability according to the knowledge of permutation and combination [5]. Different motion models are shown in Figure 1(c) to Figure 1(f).

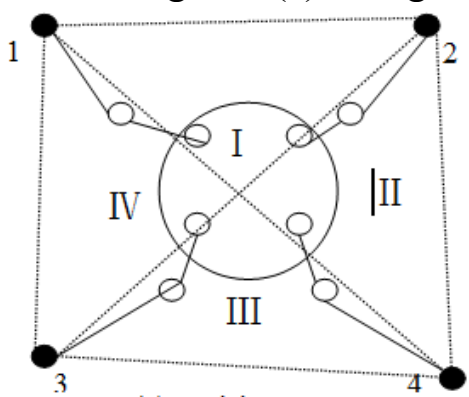

(a) Model Structure

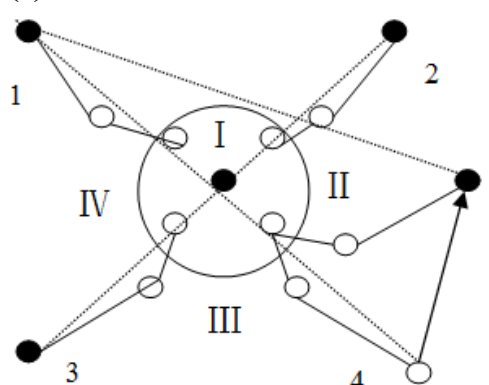

(b) Motion Model

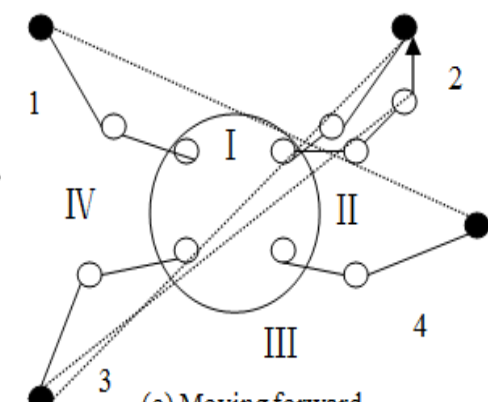

(c) Moving forward
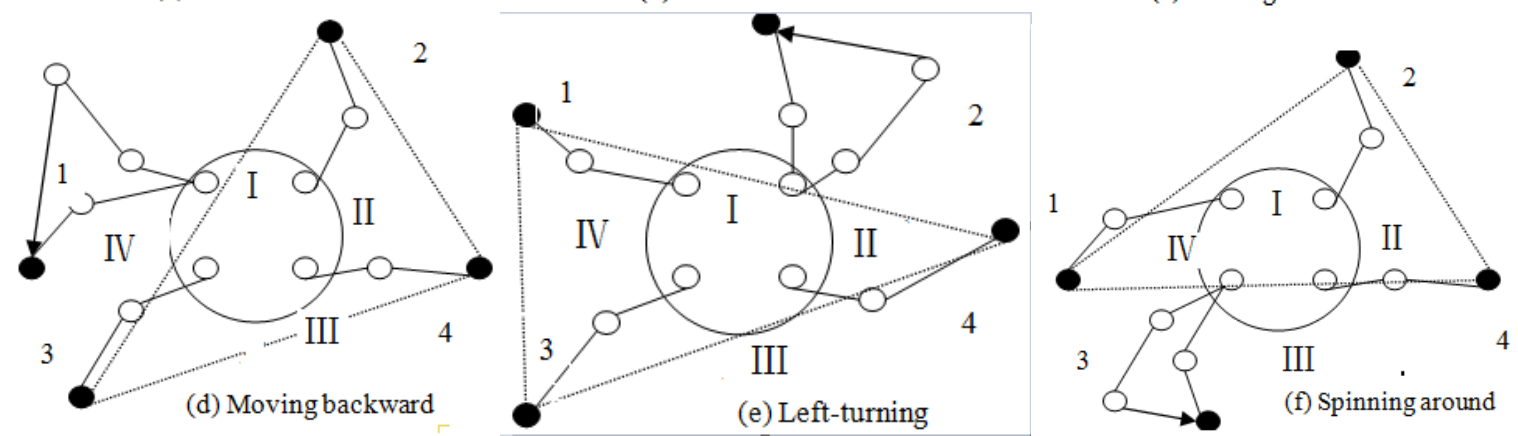

Figure 1 Model of quadruped robot

\section{Design of Hardware of Control Circuit}

The real-time control of gait planning and selection are realized with microprocessor. Models of each joint are analogue with steering gear [6]. With the driving of peripheral circuits, central processor can realize the control of each joint. In consider of the larger voltage difference caused at the moment of motor start and parking, DC motors controlled by PWM are applied to ensure the stable operation. Regulated power supply is designed to reduce the voltage vibration and improve operation stability.

The main function of quadruped robot main controller is to drive and control the rotation of the steering gear, and realize the conversation of PWM signal into the position signal of each joint [6]. Main controller mainly produce multiplex PWM signal to realize the control of robot joints, produce various types of driving signal according to the requirements of various conditions to eventually achieve the goal of the robot posture control. PWM signal are generally generated with a timer [7]. When more groups PWM are needed, a lot of resources in the main controlled are consumed. Therefore the main control chip must have faster processing speed. ATMEGA16 produced by AT Company is selected as the control core according to the requirements of the main controller. A minimum ATMEGA16 system contains Crystal circuit, reset circuit and power circuit.

ATMEGA16 has high I/O driving ability. Its output current can achieve 20mA. Then ATMEGA16 can drive peripheral devices such as numeric displays and relays directly. In this paper the control target is to switch among these above motions of the quadruped robot and to keep the static stability. Numbers from 1 to nine displayed on the numeric display are used to represent the moving speed and the smaller the number is, the lower the moving speed is.

Mode switching is realized with auxiliary keys and is controlled by manual. Key-vibrations are eliminated by a $10 \mathrm{~ms}$ delay program. The control steering mainly consists of voltage comparator and DC Motor. The main control signals for the steering are PWM signals which satisfied PPM standard and can be used to control the steering. The working principle of the steering is as follows: First, the error between the desired position and the actual position measured by compare circuit are converted into voltage signal. Then the signal is sent to the microcontroller. The microcontroller generate PWM control signal according to the error. If the error is zero, the steering will not move. If the error is positive, the steering runs clockwise. Otherwise, the steering runs counterclockwise. The deviation 
angle of the steering is in proportional to the error. With the control of the PWM signals, the deviation angle of the steering is controlled. The control circuit of the steering is shown in Fig.2.

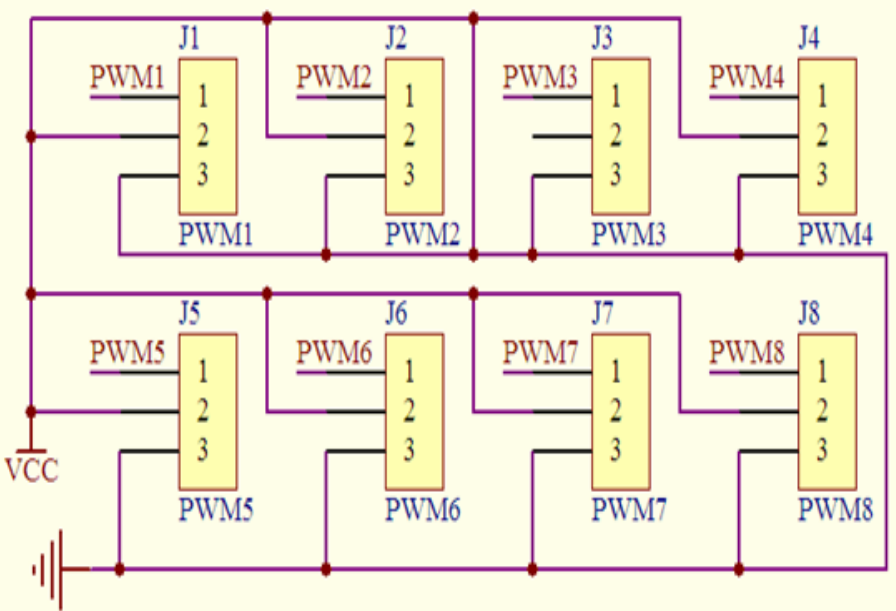

Figure 2 Control Circuit of Steering Gear Part

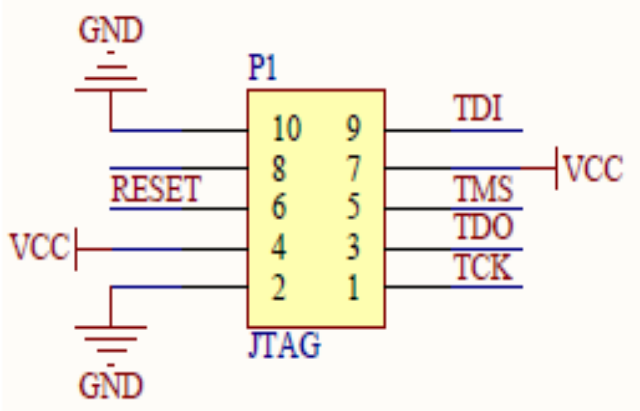

Figure 3 JTAG Interface

Fig. 3 is JTAG interface, a kind of international common standard testing interface which complies with JTAG protocol and can realize the internal processor testing and program system real-time testing. ATMEGA16 microcontroller complies with JTAG protocol and is used as the main controller. The motions of the legs are controlled by the steering which is driven by the main controller. LED digital displays the moving information and promote for further operation. Three keys are designed to realize the addition, subtraction and conformation of the speed. The main supply circuit is designed to stabilizing the external voltage $7.2 \mathrm{~V}$ at $5 \mathrm{~V}$ to insure the system voltage while the steering is working.

The system block diagram designed is shown in Fig.4. The main function of the camera is to sample the images. The N703 router is used to transform the large quantity image information at a high speed to the main microcontroller. Therefore the video information can be displayed on the screen of the PC or mobile phone and people can see the information in time.

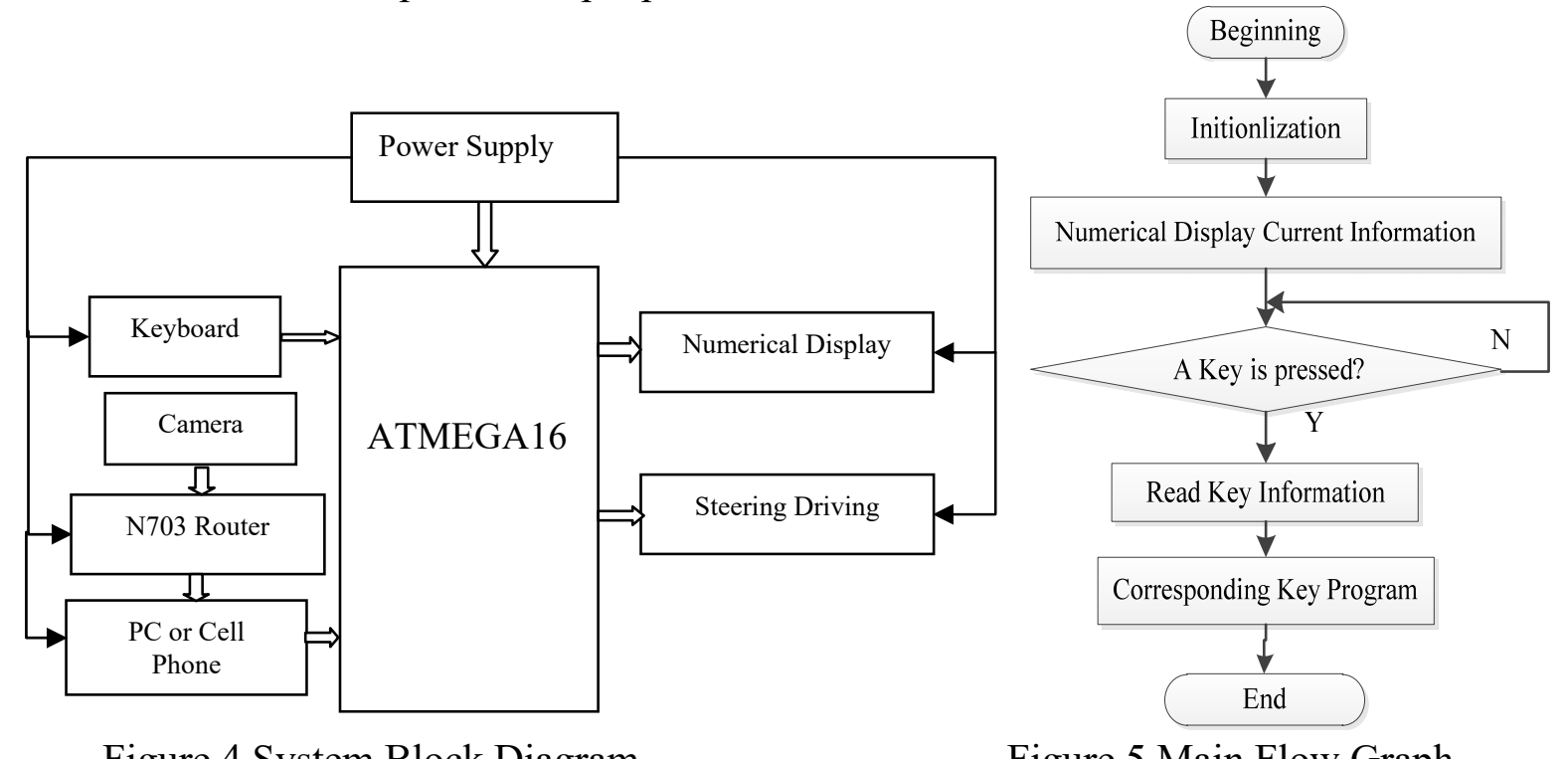

Figure 4 System Block Diagram

Figure 5 Main Flow Graph

\section{Design of System Software}

The main functions of the system designed are to realize the transformation of different status, insure the stabilization of the robot and display the corresponding information to the users so that they can make further decision. Fig. 5 following is the main flow diagram of the main system. The video information from the camera is not used for control in the main chip and is not sent into the microcontroller. 
Flow Graph of Keys Control and Numeric Display. Three keys are designed for selection of different work mode. Nine modes are designed in this control system. The flow graph of the keys control is shown in Fig.6. Numeric displays are used to display the current working mode and the selecting mode. One can make decision based on the displaying information. Fig. 7 is the display flow graph.

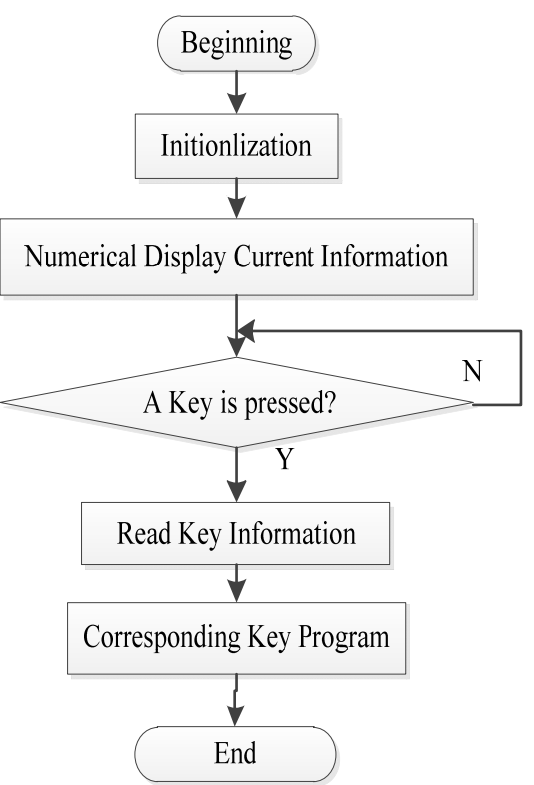

Figure 6 Flow Graph of Keys Control

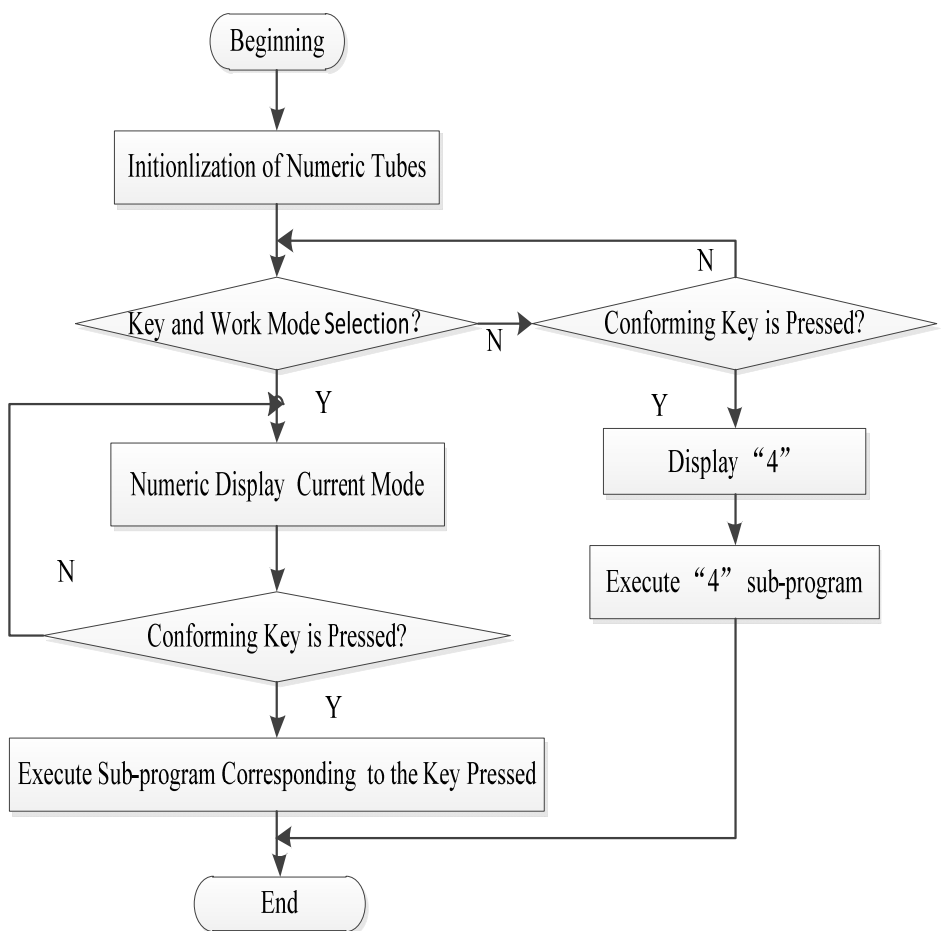

Figure 7 Flow Graph of Numeric Display

\section{Simulation and Experiments}

With the continuous testing of the steering signals of the steering controller with brand SHENGHUI, it is found that the $9 \mathrm{G}$ steering gear controller follows certain rules. It means it is controlled by standard PPM square wave signals which feature with a period of $20 \mathrm{~ms}$ and the everlasting time of high voltage is between $0.5 \mathrm{~ms}$ to $2.5 \mathrm{~ms}$. The steering only recognizes the square signal with high voltage which occurs between $0.5 \mathrm{~ms}$ to $2.5 \mathrm{~ms}$. At the same time other signals are eliminated. The corresponding relation between the everlasting time of the high voltage of the square wave and the deviation angle of the steering are shown in Table 1.

It is known from the table that the everlasting time is in perfect proportional to the deviation angle of the steering. Therefore, it is easy to achieve high precision in the control of steering angle. And so does the motions of the legs of the quadruped walking robot.

Table 1 corresponding relation between the everlasting time of high voltage and the deviation angle of steering

\begin{tabular}{|c|c|}
\hline $\begin{array}{c}\text { everlasting time of high voltage } \\
(\mathrm{ms})\end{array}$ & deviation angle of the steering $\left({ }^{\circ}\right)$ \\
\hline 0.5 & 0 \\
\hline 1.0 & 45 \\
\hline 1.5 & 90 \\
\hline 2.0 & 135 \\
\hline 2.5 & 180 \\
\hline
\end{tabular}




\section{Conclusions}

The structure of quadruped robot is realized with common analogue steering gear so that the cost of the designed system is greatly reduced. Using wireless router to send video information back to the client machine is easy and feasible. According the specific motion mode to set certain types of PWM driving signal can realize any kind motion control as well as reducing the difficulties of system design. In addition, that the inexpensive and high reliable master control chip ATMEGA16 can assure low design cost and reliability of operating. System testing shows that the designed quadruped robot can achieve all kinds of motion control such as lying down, walk, the trot, the gallop at low speed etc. But there are still some problems such as low obstacle avoiding ability, poor loading capability and poor continuous moving ability. All these problems can be solved by improvement of control algorithm and enhancement of software design.

\section{Acknowledgements}

This work was financially supported by Fundamental Research Funds for the Central Universities of Northwest University for Nationalities (Grant No. 31920150008) and by National Natural Science Foundation (NNSF) of China (Grants No. 61203189 \& No. 61463046)). The authors also gratefully acknowledge the useful suggestions from Engineer Xin HUANG who has helped to improve the system hardware and software design.

\section{References}

[1] Huai Chuangfeng, Fang Yuefa, Zhang Ketao, Robust Controller Design for a Quadruped Walking Robot, Proceedings of the 8th International Conference on Frontiers of Design and Manufacturing, 2008.

[2] Lei Sun, Biomimetic Control and Behavior Evolution of Quadruped Robot[D], University of science and technology of China, 2008.

[3] Fukuoka Y, Kimura H, Coben A.H. Adaptive Dynamic Walking of a Quadruped Robot on Irregular Terrain Based on Biological Concepts [J], 2003.

[4] Matthew D Berkemeier. Modeling of the Dynamics of Quadruped Running [J], 1998.

[5] Yasuhiro Fukuoka. Adaptive Dynamic Walking of a Quadruped Robot Tekken' on Irregular Terrain Using a Neural System Model, Proceeding IEEE Robotics and Automation, 2003

[6] Caixia Wang, Minghua Liu and Cai Ning, Drive Control of a Class of Scan Systems [C], 2013.

[7] Xing Zhang and Weichong Zhang. PWM Rectifier and Its Control [M], 2001. 